\title{
An Elegant Perturbation Iteration Algorithm for the Lane-Emden Equation
}

\author{
M. Khalid \\ Department of Mathematical Sciences \\ Federal Urdu University of Arts, Sciences \& Techonology \\ University Road, Karachi-75300, Pakistan \\ Javed Khan \\ Department of Mathematical Sciences \\ Federal Urdu University of Arts, Sciences \& Technology \\ University Road, Karachi-75300, Pakistan
}

\author{
Mariam Sultana \\ Department of Mathematical Sciences \\ Federal Urdu University of Arts, Sciences \& Technology \\ University Road, Karachi-75300, Pakistan \\ Muhammad Shoaib \\ Department of Physics \\ Federal Urdu University of Arts, Sciences \& Technology \\ University Road, Karachi-75300, Pakistan
}

\begin{abstract}
An all new technique has been devised to solve non-linear LaneEmden type equations. This novel technique is based on the Perturbation Iteration Algorithm. In this paper, a few examples are presented for the illustration of the power and wide usability of the proposed method. Moreover, a compare and contrast with the actual solution is provided. It has been evaluated that by employment of this method, the construction of perturbation solutions converging swiftly to the true solutions usually becomes easy, by giving us room to exactly demonstrate how $\epsilon$-terms influence linearized equations. This swift convergence of the method gives solutions that are accurate quantitatively through relatively little iteration.
\end{abstract}

\section{Keywords}

Lane-Emden Equation, Second-order Initial Value Problems, Perturbation Iteration Method, Numerical Solution, Fast Convergence.

\section{INTRODUCTION}

The Lane-Emden type equations are non-linear differential equations exist on a semi-infinite domain; the former lie under the category of singular initial value problems. These types of equations discuss the variation of temperature in a gas cloud of spherical shape taking into account the mutual attraction between molecules and conditional on the laws of classical thermodynamics. The polytrophic theory of stars essentially emerges from thermodynamic considerations that take into account the issue of energy transport, by considering the transfer of material among different stages of the star. The Lane-Emden type equations are the main equations in stellar structure's theory and have consequently been the subject of a great number of papers. The Lane-Emden equation is generally presented as:

$$
y^{\prime \prime}(x)+\frac{m}{x} y^{\prime}(x)+f(x, y)=g(x) ; \quad 0<x \leq 1 ; \quad m \geq 0
$$

with the initial conditions $y(0)=A$ and $y^{\prime}(0)=B$; where $f(x, y)$ is a continuous real-value function and $g(x)$ is an analytical function. Eq. (1) was employed to demonstrate multiple types of phe- nomena in physics, mathematics, and astrophysics; including the thermal behavior of a spherical cloud of gas, the theory of stellar structure, isothermal gas sphere, and the theory of thermionic currents [4 21]. The solution of the Lane-Emden type, and of all those corresponding to a variety of non-linear problems in quantum mechanics, becomes difficult numerically because there being a singular point at the origin. Bender et al. [2] introduced an entirely new perturbation technique based on an artificial parameter $\delta$; this technique is also referred to as the $\delta$-method. El-Gebeily and O'Regan [5] used the quasi linearization approach to solve the Lane-Emden type equations. The technique reckons the solution of a non-linear differential equation by taking into account the nonlinear terms as a perturbation close to the linear ones, It is different from other perturbation theories as it does not rely on the presence of a small parameter. Numerical solutions to the problems under question were introduced by authors of [22, 24] by using the Adomian method which offers solution in the form of convergent series.

Nouh [11] step up the convergence by employing an Euler-Abel transformation and Pade approximation on a power series solution of the Lane-Emden type equation. Mandelzweig and Tabakin [9] discussed Bellman and Kalaba's quasi linearization method. While Ramos [19] made use of a piecewise linearization technique. Bozkhov and Gilli Martins [3] and later Momoniat and Harley [10] used the Lie Group method effectively to generalized Lane-Emden equations. Goenner and Havas [6] also investigate the exact solutions of generalized Lane-Emden equation of the first kind . Liao [8] explained solution of Lane-Emden equation by using the Homotopy Analysis Method. Ozis and Yildirim [12,13] explored the solutions of Lane-Emden type equation by using Homotopy Perturbation and Variational Iteration Method. Parand et al. [15-18] put forward three numerical techniques to solve Lane-Emden. Their approach depended on the Rational Chebyshev, Rational Legendre Tau, and Hermite function collocation methods. He [7] obtained an approximate solution by applying a variational approach which employs a semi-inverse method. Ramos [20] introduced a series approach to the Lane-Emden equation and gave the comparison with Homotopy Perturbation Method. 
In this research, Perturbation Iteration Algorithm will be introduced for analytical solution of Lane-Emden equation. In Section 2 of this paper, the Perturbation Iteration Algorithm for this type of equations is introduced. Then, in Section 3, the suggested method is applied to some Lane-Emden equations, and a comparison is made with existing exact or analytic solutions. Finally, a brief discussion with conclusion is presented in section 4 .

\section{PERTURBATION ITERATION ALGORITHM (PIA)}

An iteration method novel in its ways, known as the "perturbationiteration method" that differs from prior published iterationperturbation methods, has been introduced recently Pakdemirli and Aksoy [14]. This technique uses a blend of Taylor series and perturbation expansions to build a scheme for iteration. Authors in [1 14$]$ introduced a single correction term in the perturbation expansion and correction terms in the Taylor Series expansion for first derivatives only, i.e. $n=1, m=1$. The algorithm is labeled $P I A(1,1)$. Let us take into consideration a general second order differential equation,

$$
G\left(y^{\prime \prime}, y^{\prime}, y, \epsilon\right)=0
$$

with $y=y(t)$ and $\epsilon$ the perturbation parameter. Only one correction term is taken in the perturbation expansion

$$
y_{n+1}=y_{n}+\epsilon\left(y_{c}\right)_{n}
$$

Upon substitution of 3 into 2 and expanding in a Taylor series with first derivatives only yields

$$
\begin{aligned}
& G\left(y_{n}^{\prime \prime}, y_{n}^{\prime}, y_{n}, 0\right)+G_{y}\left(y_{n}^{\prime \prime}, y_{n}^{\prime}, y_{n}, 0\right) \epsilon\left(y_{c}\right)_{n}+ \\
& G_{y^{\prime}}\left(y_{n}^{\prime \prime}, y_{n}^{\prime}, y_{n}, 0\right) \epsilon\left(y_{c}^{\prime}\right)+G_{y^{\prime \prime}}\left(y_{n}^{\prime \prime}, y_{n}^{\prime}, y_{n}, 0\right) \epsilon\left(y_{c}^{\prime \prime}\right)_{n}+ \\
& G_{\epsilon}\left(y_{n}^{\prime \prime}, y_{n}^{\prime}, y_{n}, 0\right) \epsilon=0
\end{aligned}
$$

where $G_{y}=\frac{\partial G}{\partial y}, G_{y^{\prime}}=\frac{\partial G}{\partial y^{\prime}}, G_{y^{\prime \prime}}=\frac{\partial G}{\partial y^{\prime \prime}}, G_{\epsilon}=\frac{\partial G}{\partial \epsilon}$. Reorganizing the Eq.(??) and simplification gives

$$
\left(y_{c}^{\prime \prime}\right)_{n}+\frac{G_{y^{\prime}}}{G_{y^{\prime \prime}}}\left(y_{c}^{\prime}\right)_{n}+\frac{G_{y}}{G_{y^{\prime \prime}}}\left(y_{c}\right)_{n}=-\frac{G_{\epsilon}+\frac{G}{\epsilon}}{G_{y^{\prime \prime}}}
$$

Taking into account the fact that all derivatives are calculated at $\epsilon=0$, it is easily determined that the given is a variable coefficient second order linear differential equation. Starting with an initial guess $y_{10}$, first $\left(y_{c}\right)_{\circ}$ is computed from Eq. (5) and then replace into Eq.(2) to calculate $y_{11}$. The iteration process is then repeated using Eq. (5) and Eq. (3) until an acceptable result is found. Consider that in the case of a marginally more general algorithm, $n$ number of correction terms in place of a single one can be used in expansion Eq. (3) that would then become a PIA $(n, m)$ algorithm. More so, this algorithm can be generalized to a differential equation system encompassing derivatives of an arbitrary order. In this paper, however, authors of this paper only take into account a case $n=m=1$ for simplicity's sake as more algebra is used in the construction of iteration solutions for $P I A(1,2)$ and $P I A(1,3)$ as evaluated to $P I A(1,1)$. The percentage error, which will be calculated in next section, is defined as

$$
\% \text { Error }=\left|\frac{\text { Exact Solution }- \text { Numerical Solution }}{\text { Exact Solution }}\right| \times 100
$$

Note that all computations pertaining to this paper have been conducted through the well-known symbolic software "Mathematica $9.0 "$

\section{NUMERICAL EXAMPLES}

In order to show the effectiveness of Perturbation Iteration Algorithm for solving the Lane-Emden equation,some numerical examples has been presented.

\subsection{Example 1}

Let look into the following non-linear Lane-Emden equation:

$$
u^{\prime \prime}(x)+\frac{2}{x} u^{\prime}(x)-\left(4 x^{2}+6\right) u(x)=0
$$

subject to the initial conditions, $u(0)=1 ; u^{\prime}(0)=0$, with exact solution $u(x)=e^{x^{2}}$. Rewrite Eq. 77 in the following form

$$
F\left(u^{\prime \prime}, u^{\prime}, u, x, \epsilon\right)=u^{\prime \prime}+\frac{2}{x} u^{\prime}-\epsilon\left(4 x^{2}+6\right) u=0
$$

where $\epsilon$ is an artificially introduced small parameter. Re-organizing the Eq. 87 yields;

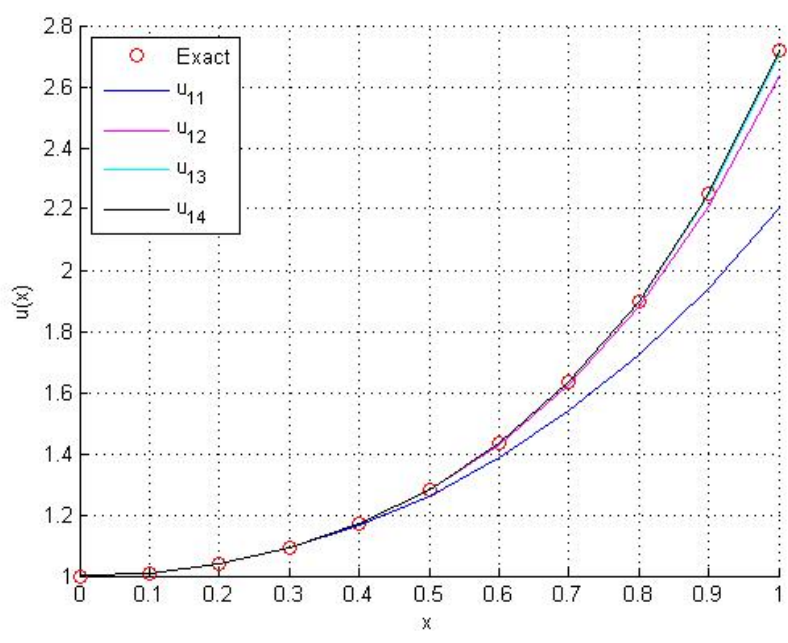

Fig. 1. Comparison of solution obtained by $P I A(1,1)$ with exact solution of Example 1

$$
x^{2} \ddot{u}^{c}+2 x \dot{u}^{c}=\frac{-x^{2} \ddot{u}-2 x \dot{u}}{\epsilon}+\left(6 x^{2}+4 x^{4}\right) u
$$

On calculating the relevant terms and setting $\epsilon=1$. The initial test function has opted for $u_{10}=1$. The approximate solution at each steps is

$$
\begin{gathered}
u_{11}=1+x^{2}+\frac{1}{5} x^{4} \\
u_{12}=1+x^{2}+\frac{1}{2} x^{4}+\frac{13}{105} x^{6}+\frac{1}{90} x^{8} \\
u_{13}=1+x^{2}+\frac{1}{2} x^{4}+\frac{1}{6} x^{6}+\frac{4}{105} x^{8}+\frac{59}{11550} x^{10}+\frac{1}{3510} x^{12} \\
u_{14}=1+x^{2}+\frac{1}{2} x^{4}+\frac{1}{6} x^{6}+\frac{1}{24} x^{8}+\frac{47}{5775} x^{10}+\frac{151}{128700} x^{12}+ \\
\frac{4987}{47297250} x^{14}+\frac{1}{238680} x^{16}
\end{gathered}
$$


Table 1. Percentage error of Example 1 with $P I A(1,1)$

$\%$ Error in \% Error in \% Error in \% Error in $\%$ Error in

\begin{tabular}{rrrrrr}
$x$ & $u_{11}$ & $u_{12}$ & $u_{13}$ & $u_{14}$ & $u_{15}$ \\
\hline
\end{tabular}

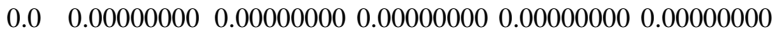

$\begin{array}{llllllll}0.1 & 0.00298669 & 0.00000427 & 0.00000000 & 0.00000000 & 0.00000000\end{array}$

$\begin{array}{lllllll}0.2 & 0.04715307 & 0.00027113 & 0.00000091 & 0.00000000 & 0.00000000\end{array}$

$\begin{array}{lllllllll}0.3 & 0.23344395 & 0.00304317 & 0.00002321 & 0.00000012 & 0.00000000\end{array}$

$\begin{array}{lllllllllll}0.4 & 0.71502286 & 0.01674168 & 0.00022989 & 0.00000207 & 0.00000001\end{array}$

$\begin{array}{llllllll}0.5 & 1.67640114 & 0.06210859 & 0.00135375 & 0.00001938 & 0.00000020\end{array}$

$\begin{array}{lllllll}0.6 & 3.30764262 & 0.17904701 & 0.00572466 & 0.00012043 & 0.00000179\end{array}$

$\begin{array}{llllllll}0.7 & 5.77683532 & 0.43249094 & 0.01921812 & 0.00056306 & 0.00001167\end{array}$

$\begin{array}{lllllll}0.8 & 9.20446292 & 0.91542291 & 0.05435449 & 0.00213323 & 0.00005932\end{array}$

$\begin{array}{lllllllll}0.9 & 13.64326247 & 1.74729396 & 0.13453147 & 0.00686681 & 0.00024871\end{array}$

$\begin{array}{llllllll}1.0 & 19.06652294 & 3.06668693 & 0.29896820 & 0.01938970 & 0.00089385\end{array}$

$\%$

Mean 4.879430360 .583919140 .046764060 .002644980 .00011050 Error

Percentage errors of Example 1 is shown in Table 1, which shows the convergence of proposed method.

\subsection{Example 2}

Let examine the following non-linear Lane-Emden equation:

$$
u^{\prime \prime}(x)+\frac{2}{x} u^{\prime}(x)-u^{n}(x)=0 ; \quad 0<x \leq 1
$$

with initial conditions, $u(0)=1 ; u^{\prime}(0)=0$. Where $n \geq 0$

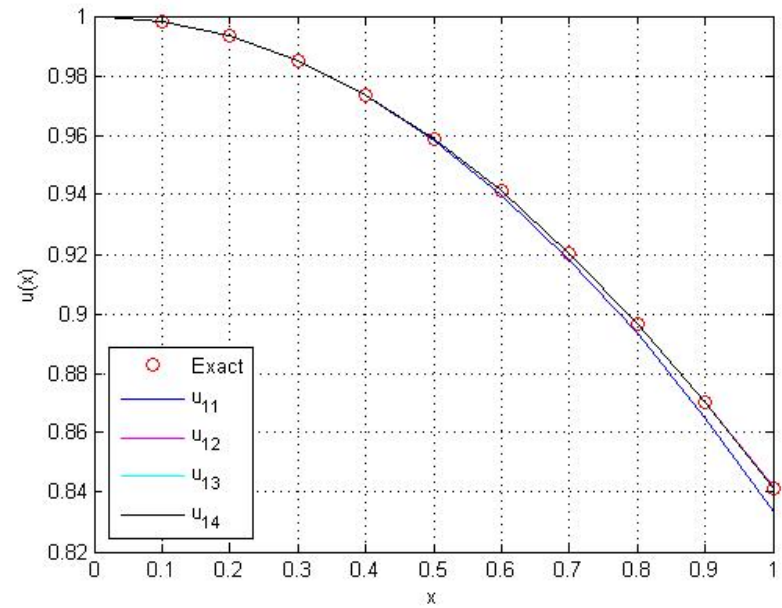

Fig. 2. Comparison of solution obtained by $P I A(1,1)$ with exact solution of Example $2(n=1)$

is constant. Substituting $n=0,1$ and 5 into Eq. (10) gives the following exact solution $u(x)=1-\frac{1}{6} x^{2}, u(x)=\frac{\sin x}{x}$ and $u(x)=\left(1+\frac{1}{3} x^{2}\right)^{-\frac{1}{2}}$ respectively. For $n=0$, solve Eq. 10 by perturbation iteration method $P I A(1,1)$ with initial function $u_{10}=1$.
Table 2. Percentage error of Example $2(n=1)$ with $P I A(1,1)$

\begin{tabular}{ccccc}
\hline & $\begin{array}{c}\text { \%Errorin } \\
x\end{array}$ & $\begin{array}{c}\% \text { Errorin } \\
u_{11}\end{array}$ & $\begin{array}{c}\% \text { Error in } \\
u_{13}\end{array}$ & $\begin{array}{c}\% \text { Error in } \\
u_{14}\end{array}$ \\
\hline 0.0 & 0.00000000000 & 0.00000000000 & 0.00000000000 & 0.00000000000 \\
0.1 & 0.00008345251 & 0.00000001987 & 0.00000000000 & 0.00000000000 \\
0.2 & 0.00134098624 & 0.00000127763 & 0.00000000070 & 0.00000000000 \\
0.3 & 0.00683765809 & 0.00001466521 & 0.00000001833 & 0.00000000001 \\
0.4 & 0.02182973067 & 0.00008329294 & 0.00000018523 & 0.00000000026 \\
0.5 & 0.05399627610 & 0.00032220418 & 0.00000112010 & 0.00000000254 \\
0.6 & 0.11378410681 & 0.00097877953 & 0.00000490234 & 0.00000001605 \\
0.7 & 0.21488914877 & 0.00251924610 & 0.00001718517 & 0.00000007665 \\
0.8 & 0.37490784102 & 0.00574922752 & 0.00005126114 & 0.00000029875 \\
0.9 & 0.61620628222 & 0.01197972026 & 0.00013529549 & 0.00000099842 \\
1.0 & 0.96707451848 & 0.02325473632 & 0.00032453164 & 0.00000295818 \\
\hline$\%$ & & & &
\end{tabular}

Mean 0.215540909170 .004082106320 .000048590920 .00000039553 Error

$$
x^{2} \ddot{u}^{c}+2 x \dot{u}^{c}=\frac{-x^{2} \ddot{u}-2 x \dot{u}}{\epsilon}+x^{2}
$$

For this equation, one may find $u_{11}=1-\frac{1}{6} x^{2}$. The exact solu-

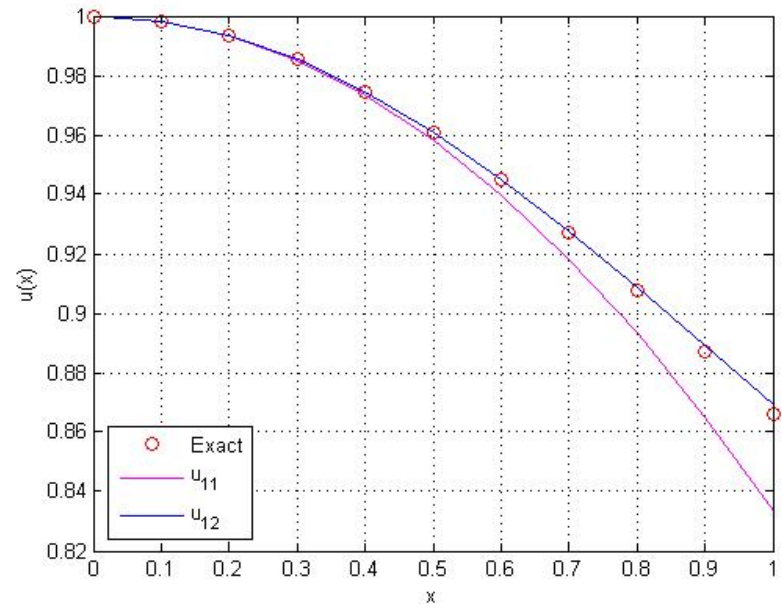

Fig. 3. Comparison of solution obtained by $P I A(1,1)$ with exact solution of Example $2(n=5)$

tion has been achieved in first iteration easily. For $n=1$, by solving Eq. 10 by perturbation iteration method with initial function $u_{10}=1$ gives

$$
x^{2} \ddot{u}^{c}+2 x \dot{u}^{c}=\frac{-x^{2} \ddot{u}-2 x \dot{u}}{\epsilon}+x^{2} u
$$

In this example, the following terms has been obtained

$$
\begin{gathered}
u_{11}=1-\frac{1}{6} x^{2} \\
u_{12}=1-\frac{1}{6} x^{2}+\frac{1}{120} x^{4}
\end{gathered}
$$


Table 3. Percentage error of Example $2(n=5)$ with $P I A(1,1)$

\begin{tabular}{ccc}
\hline & $\begin{array}{c}\text { \%Error in } \\
u_{11}\end{array}$ & $\begin{array}{c}\text { \%Error in } \\
u_{12}\end{array}$ \\
\hline 0.0 & 0.000000000000 & 0.000000000000 \\
0.1 & 0.000416204570 & 0.000000494130 \\
0.2 & 0.006637257303 & 0.000031262686 \\
0.3 & 0.033418083842 & 0.000349364222 \\
0.4 & 0.104825312101 & 0.001911428737 \\
0.5 & 0.253504192248 & 0.007047991035 \\
0.6 & 0.519750703971 & 0.020196159557 \\
0.7 & 0.950466469586 & 0.048530235984 \\
0.8 & 1.598072888488 & 0.102343140120 \\
0.9 & 2.519450658093 & 0.195069847030 \\
1.0 & 3.774955135062 & 0.342899309485 \\
\hline \% Mean Error & 0.887408809569 & 0.065307202999 \\
\hline
\end{tabular}

$$
\begin{gathered}
u_{13}=1-\frac{1}{6} x^{2}+\frac{1}{120} x^{4}-\frac{1}{5040} x^{6} \\
u_{14}=1-\frac{1}{6} x^{2}+\frac{1}{120} x^{4}-\frac{1}{5040} x^{6}+\frac{1}{362880} x^{8}
\end{gathered}
$$

which is very close to exact solution. Percentage errors of Example 2 (for $n=2$ ) are shown in Table 2. For $n=5$ in Eq. 10 and solve it by $\operatorname{PIA}(1,1)$, trial solution became $u_{10}=1$.

$$
x^{2} \ddot{u}^{c}+2 x \dot{u}^{c}=\frac{-x^{2} \ddot{u}-2 x \dot{u}}{\epsilon}+x^{2} u^{5}
$$

Other approximation solutions are

$$
u_{11}=1-\frac{1}{6} x^{2}
$$

$u_{12}=1-\frac{1}{6} x^{2}+\frac{1}{24} x^{4}-\frac{5}{756} x^{6}+\frac{5}{7776} x^{8}-\frac{1}{28512} x^{10}+\frac{1}{1213056} x^{2}$

The required accuracy has been achieved easily in second iteration [see Table 3]

\subsection{Example 3}

Consider the following non-linear Lane-Emden equation:

$$
u^{\prime \prime}(x)+\frac{8}{x} u^{\prime}(x)+x u(x)=x^{5}-x^{4}+44 x^{2}-30 x ; 0<x \leq 1
$$

Subject to the initial conditions, $u(0)=0 ; u^{\prime}(0)=0$, having the analytical solution $u(x)=x^{4}-x^{3}$. Then Eq. 14 takes the simplified form

$$
x^{2} \ddot{u}^{c}+8 x \dot{u}^{c}=\frac{-x^{3} u+x^{7}-x^{6}+44 x^{4}-30 x^{3}}{\epsilon}-x^{2} \ddot{u}-8 x \dot{u}
$$

For the initial assumed function, one may take $u_{10}=x^{2}$. By using $P I A(1,1)$, the approximate solution at each steps is

$$
\begin{gathered}
u_{11}=-x^{3}+x^{4}-\frac{1}{60} x^{5}-\frac{1}{78} x^{6}+\frac{1}{98} x^{7} \\
u_{12}=-x^{3}+x^{4}+\frac{1}{7200} x^{8}+\frac{1}{11232} x^{9}-\frac{1}{16660} x^{10} \\
u_{13}=-x^{3}+x^{4}-\frac{1}{1425600} x^{11}-\frac{1}{2560896} x^{12}+\frac{1}{4331600} x^{13}
\end{gathered}
$$

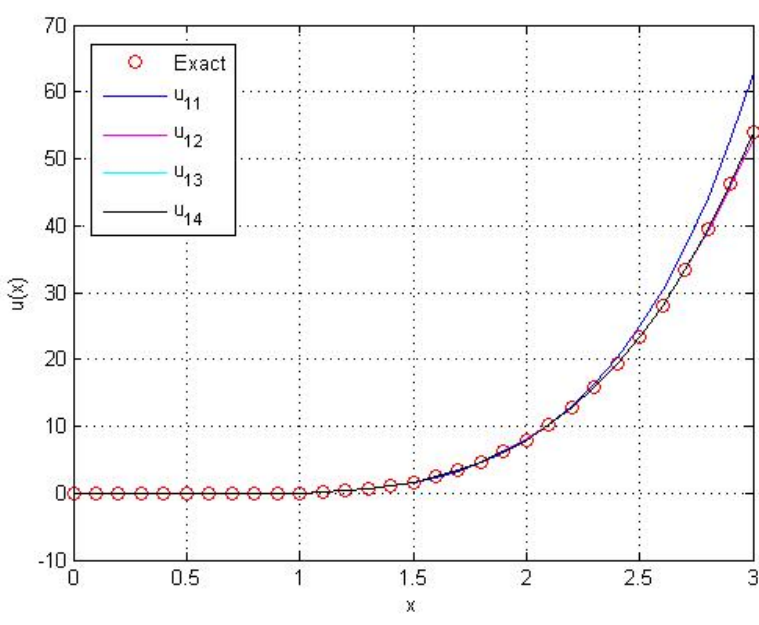

Fig. 4. Comparison of solution obtained by $P I A(1,1)$ with exact solution of Example 3

Table 4. Percentage error of Example 3 with $P I A(1,1)$

\begin{tabular}{ccccc}
\hline & $\%$ Errorin & $\begin{array}{c}\text { \%Errorin } \\
u_{12}\end{array}$ & $\begin{array}{c}\text { \%Errorin } \\
u_{13}\end{array}$ & $\begin{array}{c}\text { \%Error in } \\
u_{14}\end{array}$ \\
\hline 0.1 & 0.0198296412582 & 0.00000001635464 & 0.0000000000007 & 0.0000000000000 \\
0.2 & 0.0941130298271 & 0.0000061717679 & 0.0000000002463 & 0.0000000000000 \\
0.3 & 0.2519286835613 & 0.0000556109423 & 0.0000000074777 & 0.0000000000010 \\
0.4 & 0.5376591662304 & 0.0002814252083 & 0.0000000896440 & 0.0000000000195 \\
0.5 & 1.0262951334379 & 0.0010524909750 & 0.0000006554591 & 0.0000000002755 \\
0.6 & 1.8616954474097 & 0.0033183895096 & 0.0000035802773 & 0.0000000026031 \\
0.7 & 3.3713675213683 & 0.0096247560884 & 0.0000165580213 & 0.0000000191584 \\
0.8 & 6.5255886970166 & 0.0281310976357 & 0.0000726535898 & 0.0000001258886 \\
0.9 & 16.1512558869699 & 0.1006181061270 & 0.0003727418390 & 0.0000009234620 \\
\hline$\%$ & \multicolumn{4}{c}{}
\end{tabular}

Mean 3.31552591189770 .01589869020010 .00005180961730 .0000001190453 Error

$u_{14}=-x^{3}+x^{4}+\frac{1}{419126400} x^{14}+\frac{1}{845095680} x^{15}-\frac{1}{1594028800} x^{16}$

which is very close to analytical solution. Table 4 shows that the Perturbation Iteration Algorithm solution is very near to the exact solution.

\subsection{Example 4}

Consider the following non-linear Lane-Emden equation:

$$
u^{\prime \prime}(x)+\frac{2}{x} u^{\prime}(x)+u(x)=6+12 x+x^{2}+x^{3} ; x \geq 0
$$

subject to the initial conditions, $u(0)=0 ; u^{\prime}(0)=0$, which has the analytical solution $u(x)=x^{2}+x^{3}$. Then Eq. 16 takes the simplified

$$
x^{2} \ddot{u}^{c}+2 x \dot{u}^{c}=\frac{x^{5}+x^{4}+12 x^{3}+6 x^{2}}{\epsilon}-x^{2} u
$$

For the initial trial function, one may take $u_{10}=0$. By using $P I A(1,1)$, the successive iterations are as follow. Remember that 


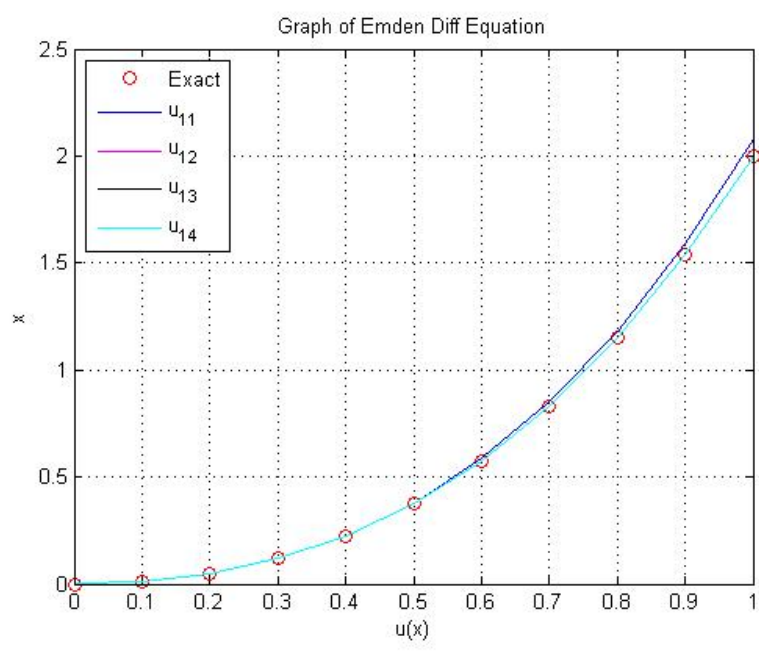

Fig. 5. Comparison of solution obtained by $P I A(1,1)$ with exact solution of Example 4

Table 5. Percentage error of Example 4 with $P I A(1,1)$

\begin{tabular}{ccccc}
\hline & $\begin{array}{c}\text { \%Errorin } \\
x\end{array}$ & $\begin{array}{c}\text { \%Error in } \\
u_{11}\end{array}$ & $\begin{array}{c}\% \text { Error in } \\
u_{13}\end{array}$ & $\begin{array}{c}\% \text { Error in } \\
u_{14}\end{array}$ \\
\hline 0.1 & 0.00303030303 & 0.00000054112 & 0.00000000006 & 0.00000000000 \\
0.2 & 0.02222222222 & 0.00001587301 & 0.00000000705 & 0.00000000000 \\
0.3 & 0.06923076923 & 0.00011126373 & 0.0000001126 & 0.00000000007 \\
0.4 & 0.15238095238 & 0.00043537415 & 0.00000077399 & 0.00000000093 \\
0.5 & 0.27777777777 & 0.00124007936 & 0.00000344466 & 0.00000000652 \\
0.6 & 0.45000000000 & 0.00289285714 & 0.00001157142 & 0.00000003155 \\
0.7 & 0.67254901960 & 0.00588480392 & 0.00003203948 & 0.00000011893 \\
0.8 & 0.94814814814 & 0.01083597883 & 0.00007705584 & 0.00000037360 \\
0.9 & 1.27894736842 & 0.01849906015 & 0.00016649154 & 0.00000102165 \\
1.0 & 1.66666666666 & 0.02976190476 & 0.00033068783 & 0.00000250521 \\
\hline$\%$ & & & &
\end{tabular}

$\begin{array}{lllll}\text { Error } & 0.50372302068 & 0.00633433965 & 0.00005656210 & 0.00000036895\end{array}$ Error

$\epsilon=1$

$$
\begin{gathered}
u_{11}=x^{2}+x^{3}+\frac{1}{20} x^{4}+\frac{1}{30} x^{5} \\
u_{12}=x^{2}+x^{3}-\frac{1}{840} x^{6}-\frac{1}{1680} x^{7} \\
u_{13}=x^{2}+x^{3}+\frac{1}{60480} x^{8}+\frac{1}{151200} x^{9} \\
u_{14}=x^{2}+x^{3}-\frac{1}{6652800} x^{10}-\frac{1}{19958400} x^{11}
\end{gathered}
$$

Table 5 shows that the Perturbation Iteration Algorithm solution is very close to the exact solution.

\subsection{Example 5}

Let us consider the following non-linear Lane-Emden equation:

$$
u^{\prime \prime}(x)+\frac{2}{x} u^{\prime}(x)+4\left[2 e^{u}+e^{\frac{u}{2}}\right]=0 ; \quad 0 \leq x \leq 1
$$

Table 6. Percentage error of Example 5 with $P I A(1,1)$

\begin{tabular}{cccc}
\hline & $\begin{array}{c}\text { \%Errorin } \\
u_{11}\end{array}$ & $\begin{array}{c}\% \text { Errorin } \\
u_{12}\end{array}$ & $\begin{array}{c}\% \text { Error in } \\
u_{13}\end{array}$ \\
\hline 0.1 & 0.499170807131 & 0.001179377880 & 0.000001626937 \\
0.2 & 1.986926764113 & 0.018352116726 & 0.000099243924 \\
0.3 & 4.435374726491 & 0.088774168783 & 0.001045030233 \\
0.4 & 7.802179276641 & 0.263725045364 & 0.005273376549 \\
0.5 & 12.035502943114 & 0.596415763377 & 0.017579287099 \\
0.6 & 17.078996221628 & 1.131785092974 & 0.044676682926 \\
0.7 & 22.875963598920 & 1.902948866105 & 0.093391201968 \\
0.8 & 29.372318986008 & 2.940648680245 & 0.167848966880 \\
0.9 & 36.518346750491 & 4.307195458556 & 0.266594016455 \\
1.0 & 44.269504088896 & 6.177848144712 & 0.381301629761 \\
\hline
\end{tabular}

\% Mean Error 17.6874284163431 .7428872714720 .097781106273

subject to the initial conditions, $u(0)=0 ; u^{\prime}(0)=0$; which has the following analytical solution $u(x)=-2 \ln \left(1+x^{2}\right)$. Then Eq. 18 takes the simplified form as

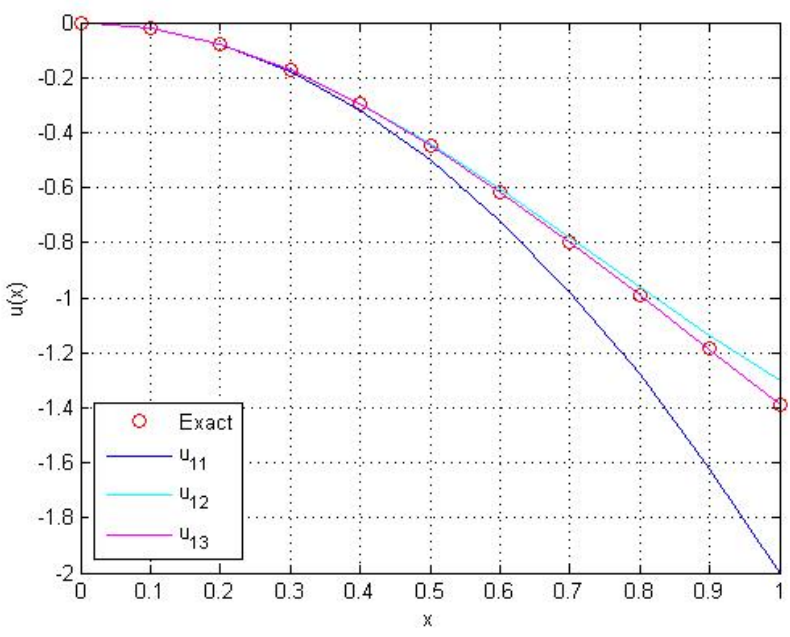

Fig. 6. Comparison of solution obtained by $P I A(1,1)$ with exact solution of Example 5

$$
\begin{aligned}
x^{2} \ddot{u}^{c}+2 x \dot{u}^{c}= & \frac{-x^{2} \ddot{u}-2 x \dot{u}}{\epsilon}-x^{2}\left(\frac{u^{5}}{10}+\frac{11 u^{4}}{32}+\frac{17 u^{3}}{12}+\right. \\
& \left.\frac{9 u^{2}}{2}+10 u+12\right)
\end{aligned}
$$

One may take $u_{10}=0$, as initial test function. By using $P I A(1,1)$, the approximate solution at each steps is

$$
u_{11}=-2 x^{2}
$$

$$
u_{12}=-2 x^{2}+x^{4}-\frac{3}{7} x^{6}+\frac{17}{108} x^{8}-\frac{1}{20} x^{10}+\frac{4}{195} x^{12}
$$




$$
\begin{aligned}
& u_{13}=-2 x^{2}+x^{4}-\frac{2}{3} x^{6}+\frac{353}{756} x^{8}-\frac{1247}{3780} x^{10}+\frac{7807}{32760} x^{12}- \\
& \frac{6138481}{36117900} x^{14}+\frac{10772227}{93562560} x^{16}-\frac{7919743}{108591840} x^{18}+ \\
& \frac{1879497509}{43688211840} x^{20}-\frac{2401561993}{101219025600} x^{22}+\frac{2391219833}{195036660000} x^{24}- \\
& \frac{64043445838231}{10764862773799680} x^{26}+\frac{56378863071233}{20752774388236800} x^{28}- \\
& \frac{111220659961319}{950742866066208000} x^{30}+\frac{899117216016931}{1889218082239488000} x^{32}- \\
& \frac{48079907405864407}{2627730605296774240000} x^{34}+\frac{132134658278053}{1987359281316864000} x^{36}- \\
& \frac{9712091484260141}{425427376820563353600} x^{38}+\frac{59248890114131}{8006519251261440000} x^{40}- \\
& \frac{226090676220211687}{99984045443051427840000} x^{42}+\frac{658887479300087}{1014972775815398400000} x^{44}- \\
& \frac{41456559628747}{23748605425461920000} x^{46}+\frac{418742503147}{9568766910044160000} x^{48}- \\
& \frac{8220115189969}{809195467010112000000} x^{50}+\frac{83383814749}{38561100859008000000} x^{52}- \\
& \frac{1941057299}{4674973811508000000} x^{54}+\frac{412463}{5815296641700000} x^{56}- \\
& \frac{3466}{63981355865625} x^{58}+\frac{8}{6614995359375} x^{60}
\end{aligned}
$$

Table 6 shows that the Perturbation Iteration Algorithm solution is very near to the exact solution.

\section{DISCUSSION OF RESULTS AND CONCLUSION}

\section{CONCLUSION}

In this research paper, the Perturbation Iteration Algorithm is introduced in order to come up with solutions for the non-linear singular Lane-Emden equation. While the merits and demerits of $P I A$ are still hazy, it proves to be a highly useful method. The Perturbation Iteration Algorithm with $n=m=1$, i.e. $P I A(1,1)$ is utilized to simplify the Lane-Emden equation. Perturbation Iteration Algorithm reduces Lane-Emden equation into a set of algebraic equations. Numerical examples demonstrate the authority and applicability of the method. The results have been compared to the analytical solution to study and assess their accuracy.

\section{Conflict of Interests}

The authors declare that there is no conflict of interests regarding the publication of this paper.

\section{Acknowledgment}

We thank the reviewers for their thorough efforts in editing our paper and highly appreciate the comments and constructive criticism that significantly contributed in improving the quality of the publication. The authors also thank Ms.Wishaal Khalid for proofreading our research paper.

\section{REFERENCES}

[1] Aksoy, Y., Pakdemirli, M. (2010) New perturbationiteration solutions for Bratu-type equations, Computers and Mathematics with Applications, Vol. 59, No. 8, pp. 2802-2808.
[2] Bender, C.M., Milton, K.A., Pinsky, S.S., Simmons, L.M. (1989) A new perturbative approach to nonlinear problems, Journal of Mathematical Physics, Vol. 30, No. 7, pp. 1447-1455.

[3] Bozhkov, Y., Gilli Martins, A.C. (2004) Lie point symmetries and exact solutions of quasilinear differential equations with critical exponents, Nonlinear Analysis: Theory, Methods \& Applications, Vol. 57, No. 5, pp. 773-793.

[4] Chandrasekhar, S. (1967) An Introduction to the Study of Stellar Structure, New York: Dover Publications, USA.

[5] El-Gebeily, M., O'Regan, D. (2007) A quasi linearization method for a class of second order singular nonlinear differential equations with non-linear boundary conditions, Nonlinear Analysis: Real World Application, Vol. 8, pp. 174-186.

[6] Goenner, H., Havas, P. (2000) Exact solutions of the generalized Lane-Emden equation, Journal of Mathematical Physics, Vol. 41, No. 10, pp. 7029-7042.

[7] He, J.H. (2003) Variational approach to the Lane-Emden equation, Applied Mathematics and Computation, Vol. 143, No. 2, pp. 539-541.

[8] Liao, S. (2003) A new analytic algorithm of Lane-Emden type equations, Applied Mathematics and Computation, Vol. 142, No. 1, pp. 1-16.

[9] Mandelzweig, V.B., Tabakin, F. (2001) Quasi linearization approach to nonlinear problems in physics with application to nonlinear ODEs, Computer Physics Communications, Vol. 141, No. 2, pp. 268-281.

[10] Momoniat, E., Harley, C. (2006) Approximate implicit solution of a Lane-Emden equation, New Astronomy, Vol. 11, pp. 520-526.

[11] Nouh, M.I. (2004) Accelerated power series solution of polytropic and isothermal gas spheres, New Astronomy, Vol. 9, No. 6, pp. 467-473.

[12] Ozis, T., Yildirim, A. (2007) Solutions of singular IVP's of Lane-Emden type by homotopy pertutbation method, Physics Letters A, Vol. 369, pp. 70-76.

[13] Ozis, T., Yildirim, A. (2009) Solutions of singular IVP's of Lane-Emden type by the variational iteration method, Nonlinear Analysis: Theory, Methods \& Applications, Vol. 70, No. 6, pp. 2480-2484.

[14] Pakdemirli, M., Aksoy, Y., Boyaci, H. (2011) A new perturbation-iteration approach for first order differential equations, Mathematical and Computational Applications, Vol. 16, No. 4, pp. 890-899.

[15] Parand, K., Razzaghi, M. (2004) Rational Chebyshev tau method for solving higher-order ordinary differential equations, International Journal of Computer Mathematics, Vol. 81, No. 1, pp. 73-80.

[16] Parand, K., Razzaghi, M. (2004) Rational Legendre approximation for solving some physical problems on semiinfinite intervals, Physica Scripta, Vol. 69, pp. 353-357.

[17] Parand, K., Dehghan, M., Rezaei, A.R., Ghaderi, S.M. (2010) An approximation algorithm for the solution of the nonlinear Lane-Emden type equations arising in astrophysics using Hermite functions collocation method, Computer Physics Communications, Vol. 181, No. 6, pp. 10961108. 
[18] Parand, K., Shahini, M., Dehghan, M. (2009) Rational Legendre pseudospectral approach for solving nonlinear differential equations of Lane-Emden type, Journal of Computational Physics, Vol. 228, No. 23, pp. 8830-8840.

[19] Ramos, J.I. (2003) Linearization methods in classical and quantum mechanics, Computer Physics Communications, Vol. 153, No. 2, pp. 199-208.

[20] Ramos, J.I. (2008) Series approach to the Lane-Emden equation and comparison with the homotopy perturbation method, Chaos, Solitons and Fractals, Vol. 38, No. 2, pp. 400-408.

[21] Richardson, O.U. (1921) The Emission of Electricity From Hot Bodies, London: Longmans Green and Company, UK.

[22] Shawagfeh, N.T. (1993) Non-perturbative approximate solution for Lane-Emden equation, Journal of Mathematical Physics, Vol. 34, No. 9, pp. 4364-4369.

[23] Wazwaz, A.M. (2001) A new algorithm for solving differential equations of Lane-Emden type, Applied Mathematics and Computation, Vol. 118, No. 2, pp. 287-310.

[24] Wazwaz, A.M. (2002) A new method for solving singular initial value problems in the second-order ordinary differential equations, Applied Mathematics and Computation, Vol. 128, No. 1, pp. 45-57. 